\title{
DITERPENÓIDES COM ESQUELETO CLEISTANTANO DE VELLOZIA AFF. CARUNCULARES MARTIUS EX SEUBERT (VELLOZIACEAE)
}

Gilson Cruz da Silva, Ligia Maria Marino Valente, Maria Lucia Patitucci, Angelo da Cunha Pinto

Instituto de Química, Departamento de Química Orgânica, Universidade Federal do Rio de Janeiro, Cidade Universitária, CT, Bloco A, Ilha do Fundão, 21949-900 Rio de Janeiro - RJ

Nanuza Luíza de Menezes

Instituto de Biologia, Departamento de Botânica, Universidade de São Paulo, 05599-970 São Paulo - SP

Recebido em 24/8/00; aceito em 29/11/00

\begin{abstract}
CLEISTANTHANE DITERPENOIDS FROM VELLOZIA AFF. CARUNCULARES MARTIUS EX SEUBERT (VELLOZIACEAE). From the hexane extract of leaves, stem and roots, of Vellozia aff. carunculares, fourteen cleistanthane diterpenoids were detected by HRGC-MS, through the analysis of their fragmentation patterns. Comparison of their MS spectra with those of authentic standards previously isolated from other species of the family Velloziaceae allowed the identification, with a high degree of confidence, of eigth known cleistanthane diterpenoids. Of these, four were isolated and identified from their MS, IR and ${ }^{1} \mathrm{H}$ and ${ }^{13} \mathrm{C}$ NMR spectra, as the known cleistantha-8,11,13-trien-7-one, cleistantha8,11,13-trien-3,7-dione, cleistantha-1,8,11,13-tetraen-3,7-dione and the first reported 6- $\alpha$-hydroxycleistantha-8,11,13-trien-7-one. The triterpenoid $\beta$-amyrin was also isolated.
\end{abstract}

Keywords: Vellozia aff. carunculares; Velloziaceae; cleistanthane diterpenoids.

\section{INTRODUÇÃO}

Velloziaceae é uma interessante família de plantas tropicais com ocorrência na África e América do Sul, expandindo-se até o Panamá. Possui cerca de 250 espécies de ervas e arbustos perenes, que crescem em solos rochosos ou arenosos, sob alta incidência solar e pouca água ${ }^{1}$.

A longevidade dessas plantas, apesar das condições inóspitas das regiões onde são encontradas, vem motivando por mais de duas décadas um estudo fitoquímico, visando encontrar as possíveis substâncias responsáveis por esse comportamento. Assim, de mais de trinta espécies brasileiras dessa família de plantas, foram isolados cerca de 180 diterpenóides inéditos, com nove diferentes tipos de esqueleto, cerca de trinta triterpenóides com seis diferentes tipos de esqueleto ${ }^{2}$ e, mais recentemente, flavonóides ${ }^{3}$.

Em um estudo sobre a bioatividade da família Velloziaceae, quarenta e seis extratos de vinte e seis espécies foram testados em bioensaio in vitro para atividade antitumoral, contra cultura de leveduras Saccharomyces cerevisiae mutantes que perderam a capacidade de regenerar o $\mathrm{DNA}^{4}$. Como resultado, oito apresentaram atividade; destes, a espécie Vellozia candida Mikan teve seus extratos ativos analisados, levando ao isolamento de uma lactona diterpênica ativa ${ }^{5}$.

Vellozia aff. carunculares, uma planta herbácea coletada em Diamantina, MG, é uma espécie quimicamente inédita, cujos extratos se mostraram inativos para a atividade antitumoral.

Este trabalho apresenta os primeiros resultados do estudo fitoquímico do extrato hexânico de Vellozia aff. carunculares. Através de seu perfil químico, traçado por meio de Cromatografia Gasosa de Alta Resolução acoplada à Espectrometria de Massas (CGAR/EM), foi evidenciada uma composição rica em di e triterpenóides. Posterior isolamento de alguns compostos e a coinjeção dos mesmos em frações brutas permitiu a identificação inequívoca de quatro diterpenóides de esqueleto cleistantano e do triterpenóide $\beta$-amirina. A utilização simultânea de técnicas instrumentais acopladas e de metodologia clássica de investigação fitoquímica, apoiada no amplo estudo fitoquímico pré-existente sobre a família Velloziaceae, revelouse uma estratégia interessante no estudo dessa espécie, permitindo até mesmo a caracterização de compostos minoritários.

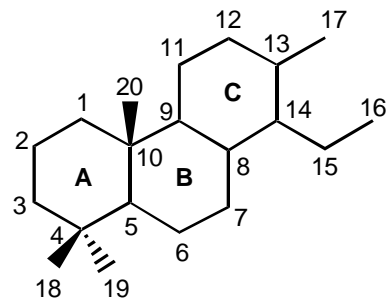

Esqueleto cleistantano

\section{RESULTADOS E DISCUSSÃO}

\section{Estudo de perfil químico}

As folhas, caule e raízes de Vellozia aff. carunculares, depois de secos, foram moídos e percolados sequiencialmente com hexano, AcOEt e $\mathrm{MeOH}$.

Inicialmente, o extrato bruto filtrado [pequena alíquota, cromatografia em coluna (CC) em sílica gel, hexano-AcOEt 70:30 como eluente] foi submetido a CGAR. Aplicando metodologia recentemente desenvolvida ${ }^{6}$, foi possível obter um perfil cromatográfico que permitiu observar a eluição dos componentes do extrato em regiões que seriam características para di e triterpenóides, e detectar a presença de um pico com tempo de retenção maior que os demais (Figura 1).

Uma alíquota de $82,0 \mathrm{mg}$ do extrato foi submetida a CC filtrante em sílica gel (gradiente hexano $\rightarrow$ AcOEt) gerando nove frações. As cinco primeiras, que se apresentaram menos polares em cromatografia em camada fina (CCF), foram analisadas por CGAR e posteriormente submetidas a CGAR/EM. A observação dos espectros de massas revelou a provável presença dos triterpenóides $\alpha$ ou $\beta$-amirona e $\alpha$ ou $\beta$-amirina, caracterizados pelos íons $\mathrm{M}^{+} 424, \mathrm{~m} / \mathrm{z}, 218$ e $203 \mathrm{e}^{+} 426, \mathrm{~m} / \mathrm{z}, 218 \mathrm{e}$ 203 , respectivamente ${ }^{7}$ e diterpenóides $\left(\mathrm{M}^{+}\right.$de 282 à 314$)$.

Estudos de fragmentografia de massas de diterpenóides com esqueleto podocarpano (com o anel $\mathrm{C}$ aromático), mostraram que o padrão de fragmentação destes compostos não é usual, mas segue um conjunto de rearranjos estreitamente relacionados ${ }^{8-11}$. 


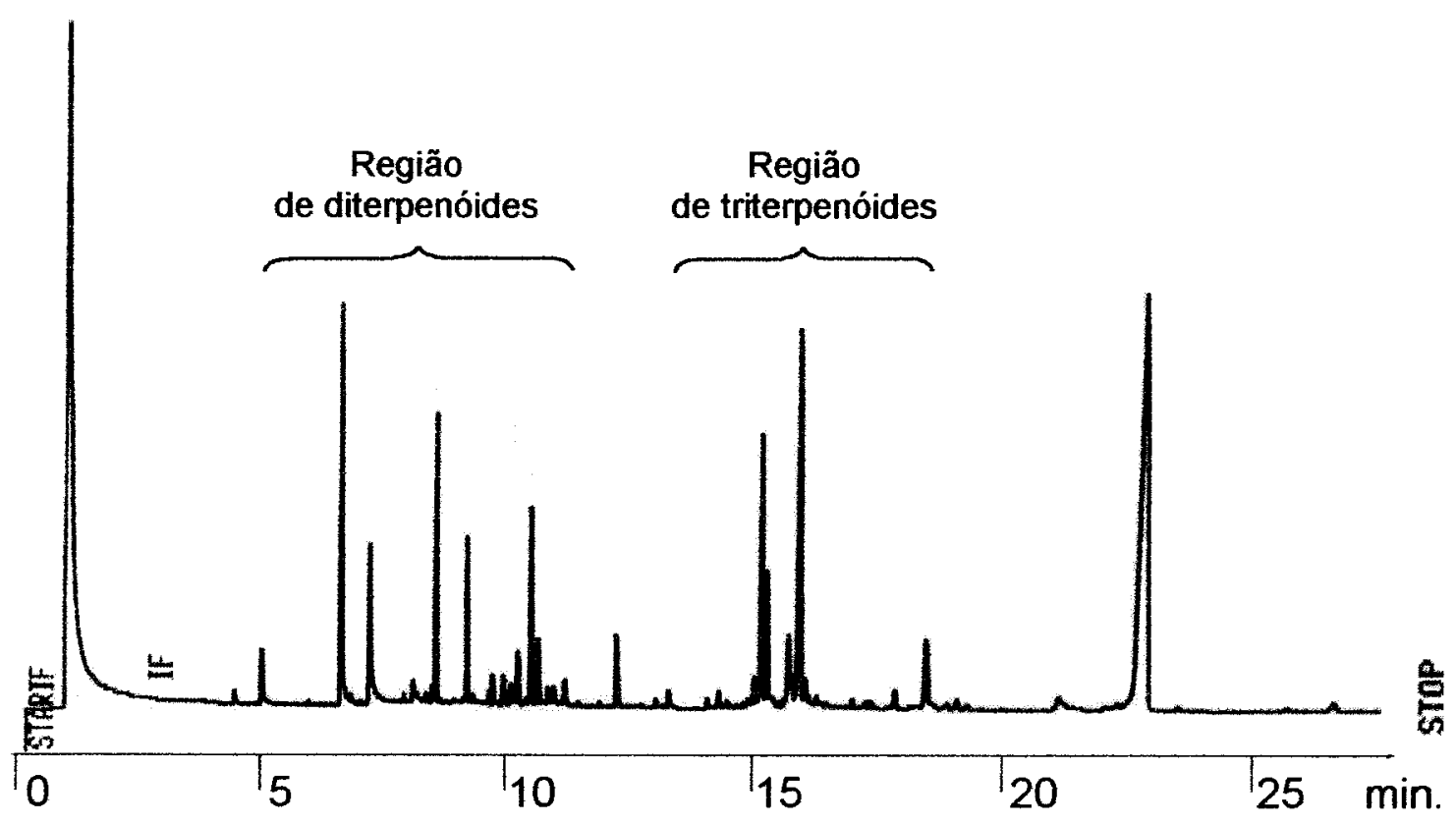

Figura 1. Cromatograma do extrato hexânico de V. aff. carunculares.

Estes estudos envolveram derivados oxigenados em diferentes posições e também o modo como a fragmentação é afetada nestes casos. As informações obtidas na fragmentação dos podocarpanos foram estendidas aos diterpenóides com esqueleto cleistantano ${ }^{12}$, em um estudo envolvendo cerca de quarenta substâncias oxigenadas nas posições $3,7,11,17,18$ e $20^{13}$. Este estudo concluiu que em geral, os cleistantanos apresentam íon molecular e íon correspondente a $\mathrm{M}^{+}-15$ bastante intensos, assim como os íons $\mathrm{M}^{+}-85, \mathrm{M}^{+}-97$ e $\mathrm{M}^{+}-111$. O espectro de massas do hidrocarboneto M1 foi utilizado como referência, para a análise da influência exercida pela presença de oxigênio em diferentes posições, nas rotas de fragmentação. O Quadro 1 apresenta as estruturas e intensidades relativas dos íons relacionados a M1. No Quadro 2 observam-se os íons característicos de cleistantanos oxigenados nos anéis A e B (posições 3 e 7) e o Quadro 3 apresenta as características dos espectros de massas de cleistantanos oxigenados no anel $\mathrm{C}$ e nas metilas $17,18,19$ e $20^{13}$.

Das frações obtidas em CC, a de número 3 apresentou um cromatograma de íons totais (Figura 2) com maior diversidade estrutural. Análise dos espectros de massas dos picos a-n (Figura 2a) revelou a presença dos fragmentos característicos para cleistantanos, conforme descrito nos Quadros 1-3, e íon molecular relativo a presença de um a três oxigênios (Tabela 1). Os picos $\mathbf{b}$ e $\mathbf{d}$ foram também observados na fração 1-2; o pico $\mathbf{f}$ foi observado em todas as frações; o pico i, na fração 4 e os picos $\mathbf{k}$ e $\mathbf{n}$ nas frações 4 e 5 .

Com base nestes dados, ficou evidenciada a presença de um total de quatorze diterpenóides com espectros de massas compatíveis com o de cleistantanos. A comparação desses espectros com os de alguns compostos anteriormente isolados de outras espécies da família Velloziaceae ${ }^{14-16}$ permitiu a proposição estrutural, com alto grau de confiabilidade, para os picos b-e, i, k e m como sendo os compostos 1-4 e 6-8 respectivamente.

\section{Isolamento de alguns diterpenóides} cleistantanos e $\beta$-amirina

Baseado nos resultados deste estudo de perfil químico, 5,0 $\mathrm{g}$ do extrato bruto foram submetidos a CC sob pressão em sílica gel (gradiente: hexano $\rightarrow \mathrm{AcOEt}$ ), sendo obtidas quarenta e seis frações, que foram analisadas por CCF e agrupadas por semelhança. As menos polares foram submetidas a CGAR/EM e recromatografadas em coluna em sílica gel e/ou CCF preparativa, levando ao isolamento dos cleistantanos $1,5,6$ e 7. Os compostos 1, 6 e $\mathbf{7}$ foram caracterizados por comparação de seus dados espectrais de Infravermelho (IV), Ressonância Magnética Nuclear de Hidrogênio $\left(\mathrm{RMN}^{1} \mathrm{H}\right)$ e Espectrometria de Massas (EM) com o de padrões autênticos.

Das frações combinadas 18-19, após acetilação com $\mathrm{Ac}_{2} \mathrm{O} /$ DMAP, foi isolado o triterpenóide $\beta$-amirina (9), na forma de seu acetato $(\mathbf{9 a})$, que foi identificado com base nos seus dados de $\mathrm{EM}$ e $\mathrm{RMN}^{1} \mathrm{H}$.

O cleistantano 1 foi isolado anteriormente de V. leptopetala ${ }^{14}$ e $V$. declinans ${ }^{15}$ e os cleistantanos 6 e 7 de $V$. leptopetala ${ }^{14}$ e $V$. piresiana ${ }^{16}$. O triterpeno 9 é de ocorrência freqüente na família Velloziaceae.

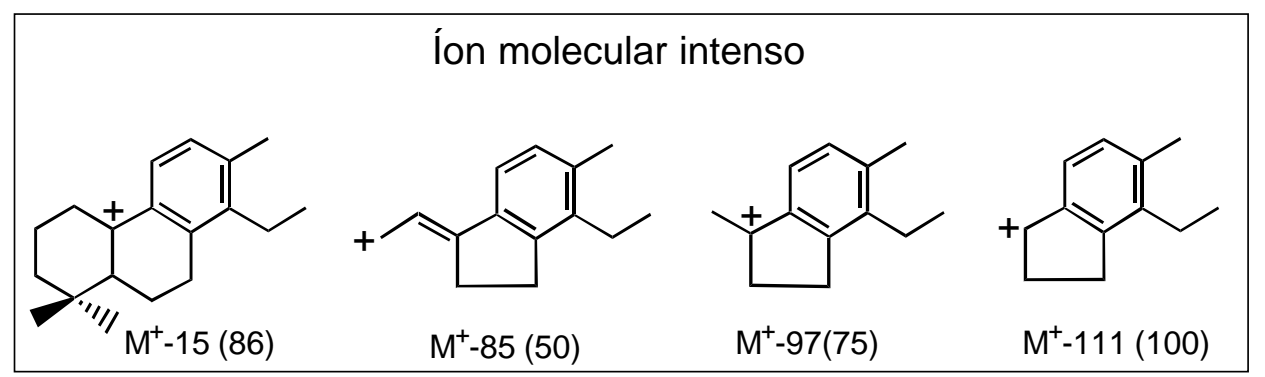

Quadro 1. Íons característicos do diterpeno cleistantano $\mathbf{M I}^{13}$. 


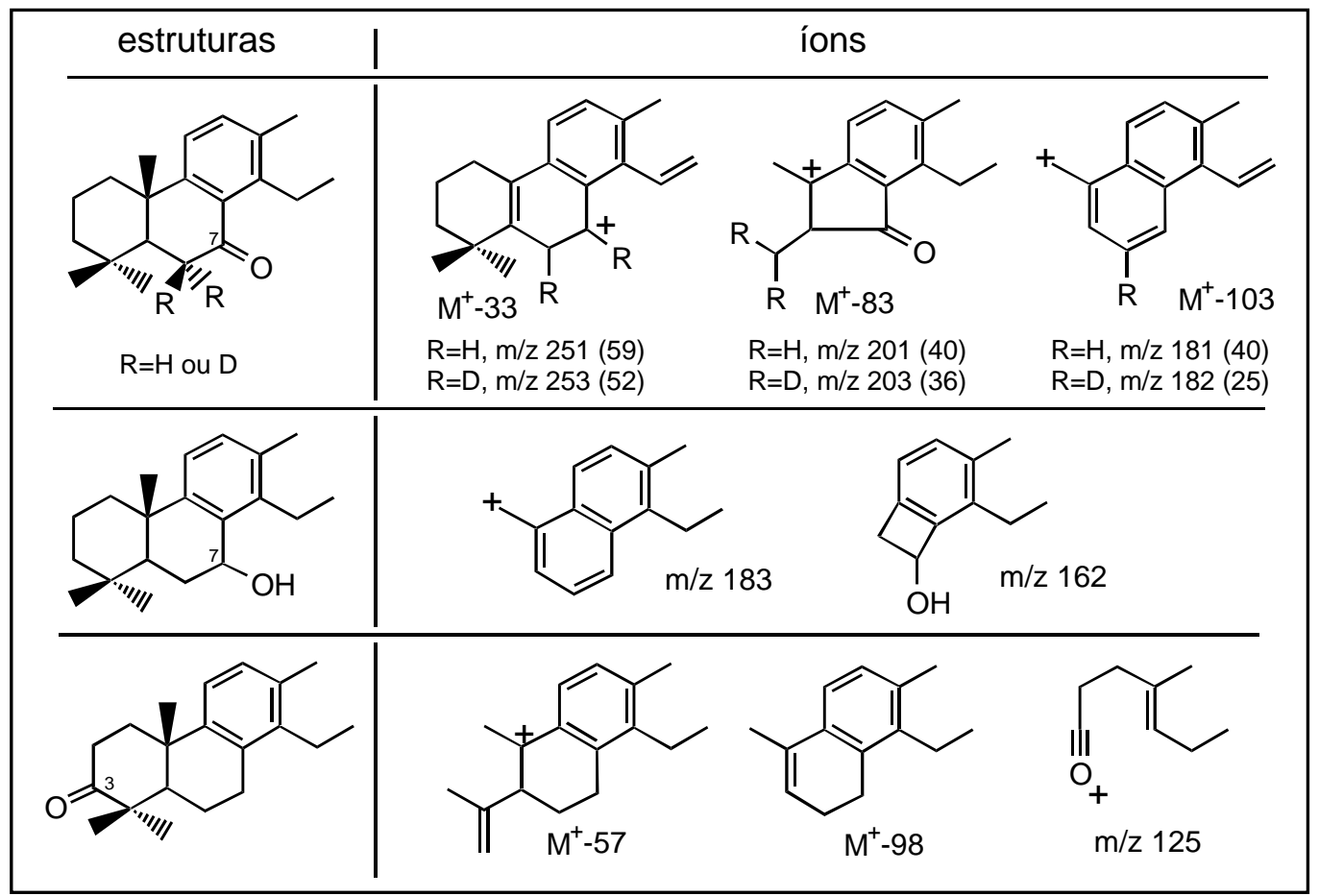

Quadro 2. Íons característicos de cleistantanos oxigenados nos anéis A e $B^{13}$

\begin{tabular}{|c|c|}
\hline posição da oxigenação & efeitos observados \\
\hline & $\begin{array}{c}\text { C-11 e C-17 - padrão de fragmentação não se altera; } \\
\text { intensidade dos íons é alterada. }\end{array}$ \\
\hline${ }_{18} \quad 11 / 19$ & $\begin{array}{l}\text { C-18 e C-19 - } \mathrm{M}^{+} \text {aparece pouco intenso; } \\
\qquad \mathrm{M}^{+}-15 \text { não aparece no espectro. } \\
\text { C-20 - intensidades de } \mathrm{M}^{+} \text {e } \mathrm{M}^{+}-15 \text { são acentuadas. }\end{array}$ \\
\hline
\end{tabular}

Quadro 3. Fragmentação característica de cleistantanos oxigenados nas posições 11 e 17-20 ${ }^{13}$

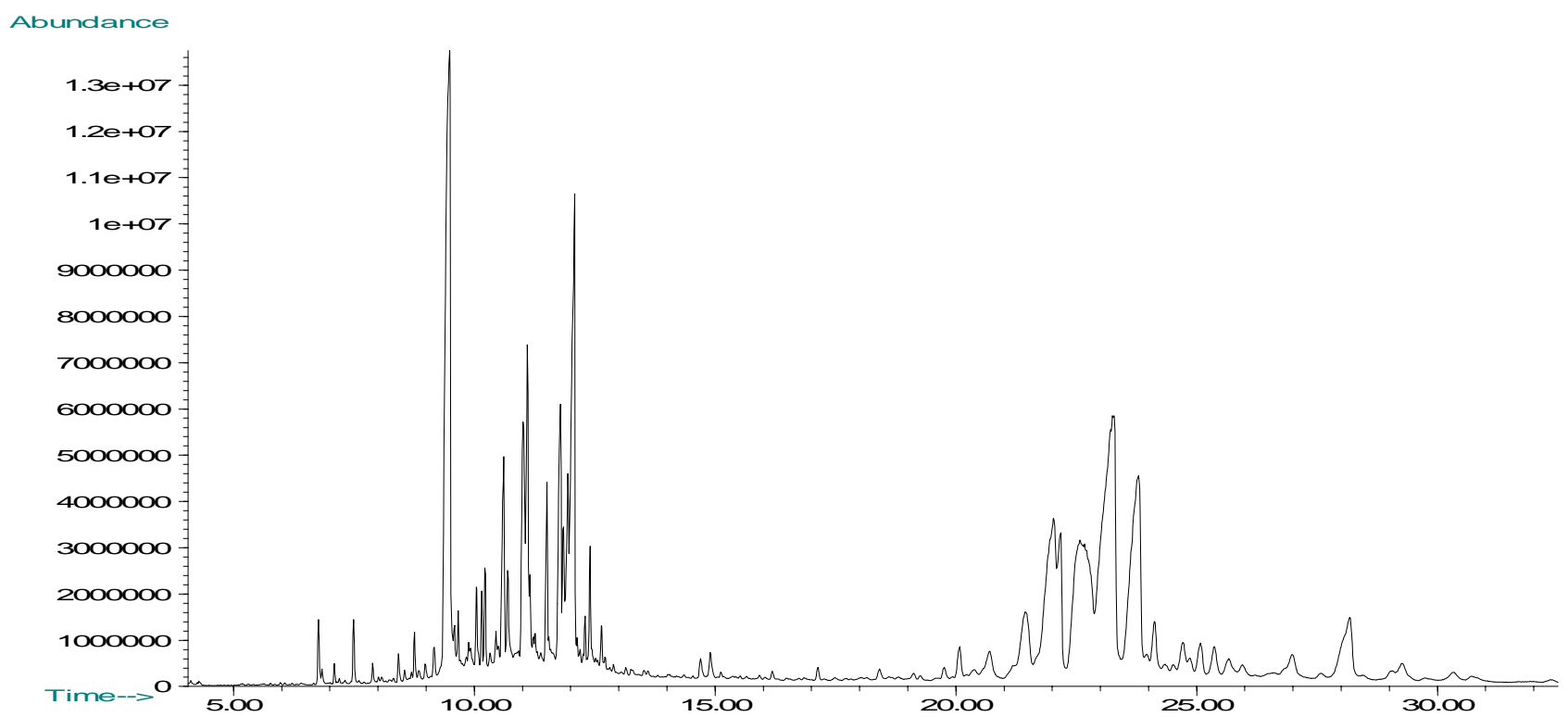

Figura 2. Cromatograma de íons totais da fração 3 do extrato hexânico de V. aff. carunculares 


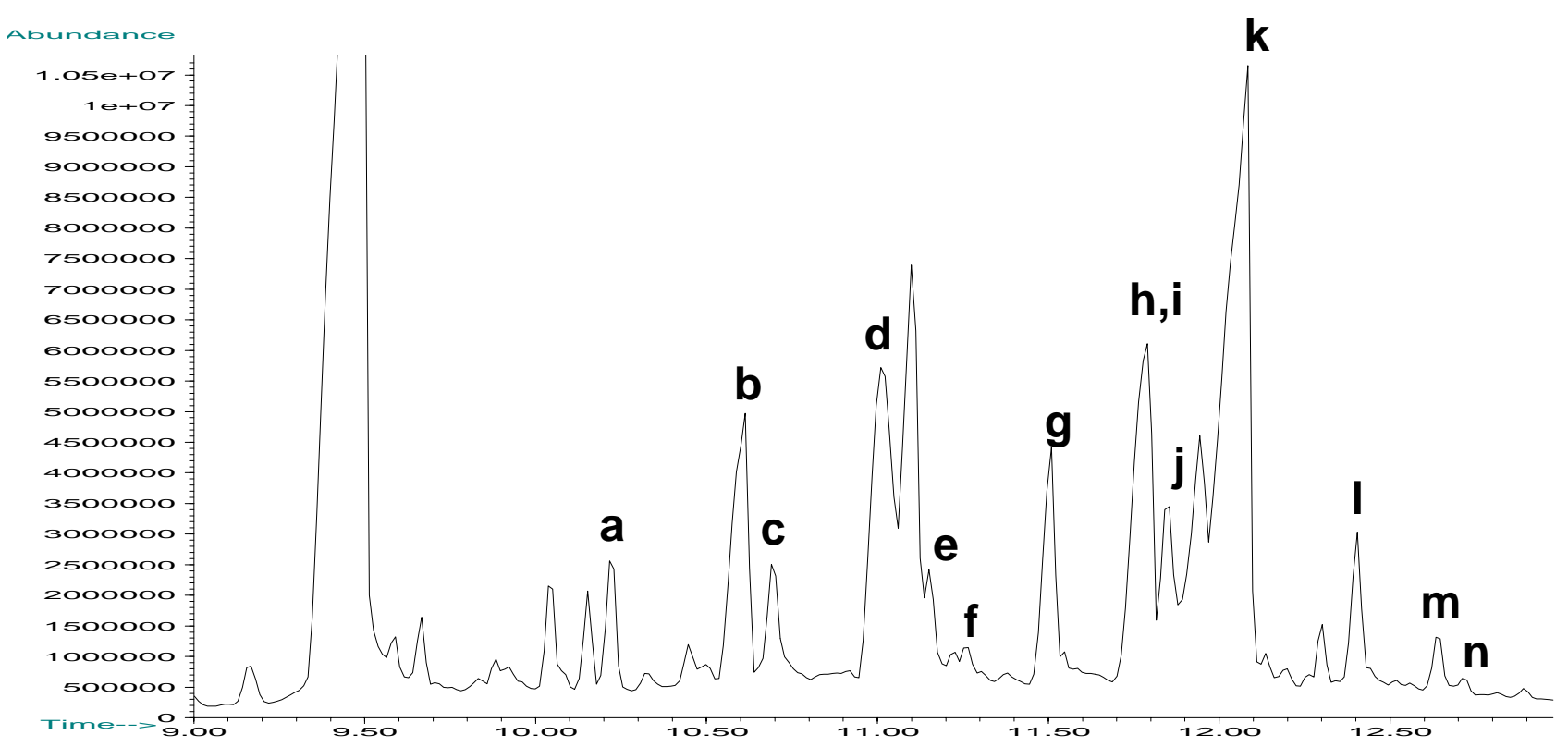

Figura 2a. Ampliação do cromatograma de ions totais - Assinalamento dos picos referentes aos diterpenóides com esqueleto cleistantano detectados na fração 3 de $V$. aff. carunculares.<smiles>[M]C1Cc2c(ccc(C)c2CC)[C@@]2(C)CCCC(C)(C)[C@@H]12</smiles><smiles>CCc1c(C)ccc2c1C(=O)[C@H](O)[C@H]1C(C)(C)CCC[C@]21C</smiles>

5<smiles>CCc1c(C)cc(O)c2c1C(=O)C[C@@H]1C(C)(C)CCC[C@]21C</smiles>

O composto 5 minoritário, mostrou por $\mathrm{EM}, \mathrm{M}^{+} 300$ e um padrão de fragmentação compatível para um diterpenóide de esqueleto cleistantano, com um átomo de oxigênio em C-7 (Tabela 1, pico f). Seu espectro de IV confirmou a presença de um grupo carbonila conjugado $\left(v 1665 \mathrm{~cm}^{-1}\right)$ além de um grupo hidroxila ( $\left.v 3367 \mathrm{~cm}^{-1}\right)$. Os espectros de $\mathrm{RMN}{ }^{1} \mathrm{H}$ e ${ }^{13} \mathrm{C}$ mostraram sinais que confirmaram o esqueleto diterpênico cleistantano: anel $\mathrm{C}$ aromático contendo os grupos metila e etila $[\delta 1,20(3 \mathrm{H}$, $\mathrm{t}, \mathrm{J}=7,0 \mathrm{~Hz}), 2,34(3 \mathrm{H}, \mathrm{s})$ e $3,08(2 \mathrm{H}, \mathrm{m})]$, dois hidrogênios aromáticos vicinais $(\delta 7,15, \mathrm{~d}, \mathrm{~J}=8,0 \mathrm{~Hz}$ e $7,29, \mathrm{~d}, \mathrm{~J}=8,0 \mathrm{~Hz})$ e quatro átomos de carbono aromáticos substituídos $(\delta$ 127,0,
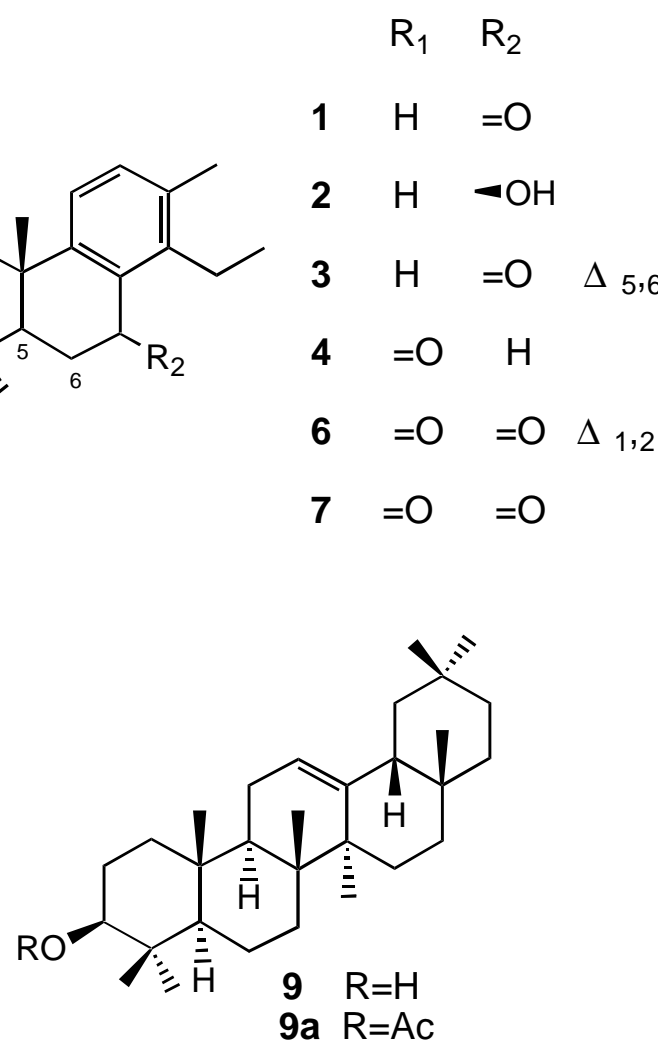

135,4, 145,4 e 154,7). Esses espectros mostraram também um grupo carbonila em $\delta 201,2$ e a presença de um grupo hidroxila secundário em posição equatorial $\left(\delta_{\mathrm{H}} 4,58,1 \mathrm{H}, \mathrm{dl}, \mathrm{J}=12,3 \mathrm{~Hz}\right.$ e $\left.\delta_{C} 74,6\right)$. Esses dados permitiram identificar o composto 5 como o diterpenóide 6- $\alpha$-hidróxi-cleistanta-8,11,13-trieno-7-ona, também isolado de Vellozia leptopetala ${ }^{13}$ e pela primeira vez descrito. Para verificar a possível desidratação do composto 5 no injetor do CG, que resultaria na formação do composto $\mathbf{3}$, o composto 5 puro foi submetido às mesmas condições de CGEM. O cromatograma resultante não demonstrou a presença de produtos de degradação. 
Tabela 1. Principais íons nos E.M. correspondentes aos picos a-n (Figura 2a)

\begin{tabular}{|c|c|c|c|c|c|c|c|c|c|c|c|c|c|c|}
\hline & $\mathbf{a}$ & b & c & d & e & f & $\mathbf{g}$ & $\mathbf{h}$ & $\mathbf{i}$ & $\mathbf{j}$ & $\mathbf{k}$ & 1 & $\mathbf{m}$ & $\mathbf{n}$ \\
\hline Frag/ $\mathrm{T}_{\mathrm{R}}(\min )^{*}$ & 10,23 & 10,63 & 10,70 & 11,02 & 11,15 & 11,28 & 11,51 & 11,73 & 11,80 & 11,83 & 12,08 & 12,40 & 12,65 & 12,73 \\
\hline $\mathrm{M}^{+}$ & $286(33)$ & $284(100)$ & $286(16)$ & $282(100)$ & $284(55)$ & $300(34)$ & $298(100)$ & $312(100)$ & $296(100)$ & $300(95)$ & $298(100)$ & $312(100)$ & $300(89)$ & 314 (100) \\
\hline $\mathrm{M}^{+}-15$ & $271(27)$ & $269(21)$ & - & $267(65)$ & 269 (100) & 285 (14) & $283(21)$ & $297(67)$ & $281(26)$ & - & $283(10)$ & $297(12)$ & $285(33)$ & - \\
\hline $\mathrm{M}^{+}-18$ & - & - & $268(70)$ & - & - & - & - & - & - & $282(12)$ & - & - & - & $296(31)$ \\
\hline $\mathrm{M}^{+}-33$ & - & $251(76)$ & $253(49)$ & - & - & 267 (33) & $265(3)$ & - & $263(21)$ & $267(25)$ & $265(29)$ & 279 (16) & $267(21)$ & $281(38)$ \\
\hline $\mathrm{M}^{+}-57$ & - & - & - & - & $227(73)$ & - & $241(42)$ & $255(7,0)$ & - & - & 241 (13) & - & - & - \\
\hline $\mathrm{M}^{+}-83$ & - & $201(29)$ & - & 199 (27) & - & - & - & - & - & - & - & $229(16)$ & $217(15)$ & - \\
\hline $\mathrm{M}^{+}-85$ & $201(22)$ & $199(53)$ & $201(7,0)$ & $197(35)$ & 199 (15) & 215 & 213 (12) & $227(68)$ & 211 & - & 213 (16) & 227 (23) & 215 (100) & - \\
\hline $\mathrm{M}^{+}-97$ & 189 (16) & $187(69)$ & $189(9)$ & 185 (13) & 187 (10) & $203(16)$ & 201 (44) & 215 & $199(56)$ & - & 201 (13) & 215 & $203(71)$ & - \\
\hline $\mathrm{M}^{+}-98$ & - & - & - & - & $186(16)$ & - & $200(32)$ & - & - & - & $200(8)$ & - & - & 216 \\
\hline $\mathrm{M}^{+}-101$ & - & - & - & - & $183(25)$ & - & - & - & - & $199(41)$ & - & - & - & 213 (16) \\
\hline $\mathrm{M}^{+}-103$ & - & 181 & - & 179 (33) & - & - & - & 209 (9) & - & - & 195 (14) & - & $197(25)$ & $211(74)$ \\
\hline $\mathrm{M}^{+}-111$ & $175(8)$ & $173(29)$ & $175(9)$ & $171(4)$ & $173(11)$ & 189 (4) & 187 (23) & - & 185 (18) & - & $187(24)$ & - & $189(52)$ & - \\
\hline $\mathrm{M}^{+}-113$ & - & - & - & - & $113(27)$ & $187(56)$ & $185(47)$ & $199(20)$ & $187(38)$ & $187(34)$ & - & - & - & 201 \\
\hline$M^{+}-127$ & - & - & - & - & 157 (33) & - & - & - & $173(60)$ & $173(53)$ & - & - & - & 187 (53) \\
\hline $\mathrm{m} / \mathrm{z} 125$ & - & - & - & - & (31) & - & - & - & - & - & (47) & - & - & - \\
\hline $\mathrm{m} / \mathrm{z} 162$ & - & - & $(65)$ & - & - & - & - & - & - & - & - & - & - & - \\
\hline $\mathrm{m} / \mathrm{z} 183$ & - & - & (100) & - & - & - & - & - & - & - & - & - & - & - \\
\hline $\mathrm{m} / \mathrm{z} 200$ & - & - & - & (23) & - & $(100)$ & (32) & $(53)$ & $(79)$ & (32) & - & $(25)$ & - & - \\
\hline Outros & $245(100)$ & 157 (15) & 211 (16) & 239 (13) & $213(25)$ & $205(51)$ & $199(51)$ & $165(35)$ & 253 (12) & $241(51)$ & $223(15)$ & 201 (17) & $201(21)$ & 225 (19) \\
\hline íons & $163(82)$ & $141(18)$ & $197(28)$ & $213(77)$ & $185(38)$ & $163(21)$ & $228(32)$ & $128(16)$ & $165(35)$ & $201(71)$ & 199 (19) & $163(14)$ & $165(18)$ & $199(22)$ \\
\hline desta- & $147(25)$ & $128(22)$ & $147(28)$ & $165(25)$ & 159 (33) & $105(22)$ & $128(30)$ & 83 (18) & 128 (19) & $183(100)$ & $181(20)$ & 115 (12) & 128 (18) & $173(31)$ \\
\hline cados & 121 & 69 (17) & 69 (18) & $152(15)$ & $69(25)$ & 69 (10) & 69 (12) & $69(40)$ & $69(16)$ & 185 & 173 & 69 (10) & $69(21)$ & 128 \\
\hline
\end{tabular}

*Tempos de retenção referentes ao cromatograma de íons totais da fração 3 do estudo de perfil químico de Vellozia aff. carunculares 


\section{Coinjeções}

Os compostos 1, 6 e 7 majoritários, isolados neste trabalho, foram coinjetados nas frações 1-2 e 3, onde foram inicialmente detectados. Foram observadas coeluições com aumento de cerca de $30 \%$ nas áreas dos picos cromatográficos correspondentes.

\section{PARTE EXPERIMENTAL}

\section{Procedimentos experimentais gerais}

$\mathrm{O}$ espectro no UV do composto 5 foi feito em $\mathrm{MeOH}$ em espectrofotômetro Beckman DB-GT e sua rotação ótica em um polarímetro Perkin-Elmer modelo 241 usando $\mathrm{CHCl}_{3}$ como solvente. Os espectros de $\mathrm{RMN}{ }^{1} \mathrm{H}$ a $200 \mathrm{MHz}(\delta \mathrm{ppm}, \mathrm{J}$ em Hz$)$ foram obtidos em Espectrômetro Brüker AC 2000-A e o espectro de $\mathrm{RMN}{ }^{13} \mathrm{C}$ a $25,2 \mathrm{MHz}$ do composto 5 foi feito em um espectrômetro Varian XL-100 sempre usando $\mathrm{CDCl}_{3}$ como solvente e TMS ou solvente como padrão interno. As análises por Cromatografia Gasosa de Alta Resolução (CGAR) foram feitas em cromatógrafo HP 5790 em coluna SE-54 (fase: 1\% vinil, 5\% fenil $94 \%$ metilsilicone) de 20 metros, injetor a $270{ }^{\circ} \mathrm{C}$ e detetor FID a $290{ }^{\circ} \mathrm{C}$, vazão do gás de arraste $\left(\mathrm{H}_{2}\right)$ de $2 \mathrm{ml} \mathrm{min}^{-1}$, razão de split 1:20 e volume de injeção de $1 \mu \mathrm{l}$, utilizando como programação uma temperatura inicial de $140^{\circ} \mathrm{C}$, com rampa de aquecimento de $12^{\circ} \mathrm{C} / \mathrm{min}$ até $290^{\circ} \mathrm{C}$, e isoterma de 20 a 30 minutos nesta temperatura. Os espectros de massas foram obtidos a $70 \mathrm{eV}$ em Espectrômetro de Massas HP 5987 A. Os espectros na região do Infravermelho foram obtidos em Espectrômetro Perkin-Elmer, modelo 467 de feixe duplo, usando pastilhas de $\mathrm{KBr}$. As frequiências de absorção foram medidas em unidades de número de onda $\left(\mathrm{cm}^{-1}\right)$. As colunas cromatográficas foram desenvolvidas em sílica gel 60 (35-70 mesh para coluna filtrante e 230-400 mesh para coluna sob pressão). As análises cromatográficas em camada fina analítica foram desenvolvidas em placas pré-preparadas com sílica gel $60 \mathrm{~F}_{254}$, com detecção dos compostos sob luz UV (254 e 366 $\mathrm{nm})$ e por borrifamento de $\mathrm{CeSO}_{4} / \mathrm{H}_{2} \mathrm{SO}_{4}$ seguida de aquecimento; placas cromatográficas de camada fina preparativa foram desenvolvidas em sílica gel 60 PF com espessura de camada de 0,7 $\mathrm{mm}$, detecção sob luz UV e recuperação dos compostos usando $\mathrm{CH}_{2} \mathrm{Cl}_{2}$ ou AcOEt.

\section{Material vegetal}

A espécie Vellozia aff. carunculares utilizada neste estudo foi coletada em Diamantina, Minas Gerais, em 1993. A exsicata encontra-se depositada no herbário do Jardim Botânico da Universidade de São Paulo (São Paulo, Brasil), sob o número 0329-SPF.

\section{Isolamento dos constituintes de $\boldsymbol{V}$. aff. Carunculares}

A planta inteira (raízes, caule e folhas) seca e moída foi percolada sucessivamente com hexano $(84,2 \mathrm{~g})$, AcOEt $(57,7 \mathrm{~g})$ e $\mathrm{MeOH}(46,2 \mathrm{~g})$. Parte do extrato hexânico (5,0 g) foi submetida a $\mathrm{CC}$ e eluída com mistura de hexano/AcOEt de polaridade crescente, sendo obtidas quarenta e seis frações. Com base no perfil em CCF, três frações combinadas principais foram obtidas: $\mathrm{n}^{\text {os }} 14-17(2,4 \mathrm{~g}), 18-19(341,5 \mathrm{mg})$ e $20-21(774,2 \mathrm{mg})$. Uma porção de 1,01 g das frações $14-17$ foi recromatografada em CC sob pressão com hexano-AcOEt 95:5 como eluente, gerando 30 subfrações. Da subfração 14-16 (158,7 mg), após cristalização, obteve-se $54,1 \mathrm{mg}$ do composto 1 e da subfração 17 (13,7 mg), após CCF preparativa eluída com hexano-AcOEt 99:1, obteve-se o composto 5 (1,3 mg). Parte da fração 20-21 (99,1 mg) após duas CCF preparativas consecutivas usando hexano-AcOEt 97:3 e hexano-i-PrOH 95:5 como eluentes respectivamente, gerou $3,5 \mathrm{mg}$ do composto 7 e uma mistura dos compostos 6 e 7 (3,6 mg). Os dados de RMN ${ }^{1} \mathrm{H}$ do composto
6 foram obtidos por comparação dos dados em RMN ${ }^{1} \mathrm{H}$ da mistura dos compostos 6 e 7 com aqueles do composto 7 puro. A fração 18-19 (341,5 mg) foi recromatografada em CC sob pressão, eluindo-se com $\mathrm{CH}_{2} \mathrm{Cl}_{2}$-AcOEt 70:30, gerando 24 subfrações. Acetilação de $51,2 \mathrm{mg}$ da subfração $16 \mathrm{com} 10 \mathrm{~mL}$ de $\mathrm{Ac}_{2} \mathrm{O}$ e DMAP como catalisador (4 h., t.a.), produziu após purificação com CCF preparativa seguida de cristalização, 8,0 mg do composto $9 \mathbf{a}$.

\section{Cleistanta-8,11,13-trien-7-ona (1)}

Cristais incolores (hexano-AcOEt 10:1); p.f. $=88-91^{\circ} \mathrm{C}$; $\mathrm{RMN}^{1} \mathrm{H}\left(200 \mathrm{MHz}, \mathrm{CDCl}_{3}\right) \delta 0,90(3 \mathrm{H}, \mathrm{s}), 1,01(3 \mathrm{H}, \mathrm{s}), 1,15$ $(3 \mathrm{H}, \mathrm{s}), 1,21(3 \mathrm{H}, \mathrm{t}, J=7,3 \mathrm{~Hz}), 2,32(3 \mathrm{H}, \mathrm{s}), 2,90(2 \mathrm{H}, \mathrm{m})$, $7,08(1 \mathrm{H}, \mathrm{d}, J=8,0 \mathrm{~Hz}), 7,25(1 \mathrm{H}, \mathrm{d}, J=8,0 \mathrm{~Hz}) ; \mathrm{IV} v_{\max }$ (KBr) $\mathrm{cm}^{-1}$ 2925, 2865, 1672, 1470, 1378, 1270, 1245, 1132; EM $70 \mathrm{eV}$ (ab. rel.) m/z: Tabela 1 (letra b).

\section{6- $\alpha$-Hidróxi-cleistanta-8,11,13-trien-7-ona (5)}

UV $\lambda_{\max }(\mathrm{MeOH}) \mathrm{nm}(\log \varepsilon) 222(3,87), 257(3,85)$ e 302 $(2,29) ; \quad[\alpha]_{24}=\frac{5898546}{+29,6+30,9+39,0} \mathrm{~nm}\left(\mathrm{c}=0,84, \mathrm{CHCl}_{3}\right) ; \quad$ IV $v_{\max }(\mathrm{KBr}) \mathrm{cm}^{-1} 3367,2933,1665,1600,1445,1380,1013$, 830; $\mathrm{RMN}^{1} \mathrm{H}\left(200 \mathrm{MHz}, \mathrm{CDCl}_{3}\right) \delta 1,20(3 \mathrm{H}-16, \mathrm{t}, J=7,0$ $\mathrm{Hz}), 1,25(3 \mathrm{H}, \mathrm{s}), 1,34(6 \mathrm{H}, \mathrm{s}), 1,76(\mathrm{H}-5, \mathrm{~d}, J=12,3 \mathrm{~Hz})$, $2,34(3 \mathrm{H}-17, \mathrm{~s}), 3,08(2 \mathrm{H}-15, \mathrm{~m}), 3,83(\mathrm{OH}, \mathrm{sl}), 4,58(\mathrm{H}-6, \mathrm{dl}$, $J=12,3 \mathrm{~Hz}), 7,15(1 \mathrm{H}, \mathrm{d}, J=8,0 \mathrm{~Hz}), 7,29(1 \mathrm{H}, \mathrm{d}, J=8,0$ $\mathrm{Hz})$. RMN ${ }^{13} \mathrm{C}\left(25,2 \mathrm{MHz}, \mathrm{CDCl}_{3}\right) \delta 14,0(\mathrm{C}-16), 19,1$ (C17), 19,2 (C-2), 22,0 (C-19), 23,8 (C-15), 25,3 (C-20), 34,1 (C-4), 35,6 (C-18), 39,4 (C-1), 39,5 (C-10), 42,8 (C-3), 55,7 (C-5), 74,6 (C-6), 121,4 (C-11), 127,0 (C-8), 135,4 (C-13), 135,6 (C-12), 145,4 (C-14), 154,7 (C-9), 201,2 (C-7); EM 70 $\mathrm{eV}$ (ab. rel.) $\mathrm{m} / \mathrm{z}$ : Tabela 1 (letra f).

\section{Cleistanta-8,11,13-trien-3,7-diona (7)}

IV $v_{\max }(\mathrm{KBr}) \mathrm{cm}^{-1} 2940,2860,1710,1670,1460,1375$, 1110; $\mathrm{RMN}^{1} \mathrm{H}\left(200 \mathrm{MHz}, \mathrm{CDCl}_{3}\right) \delta 1,09(3 \mathrm{H}, \mathrm{s}), 1,20(3 \mathrm{H}, \mathrm{t}$, $J=7,4 \mathrm{~Hz}), 1,37(3 \mathrm{H}, \mathrm{s}), 1,51(3 \mathrm{H}, \mathrm{s}), 2,33(3 \mathrm{H}, \mathrm{s}), 7,04(1 \mathrm{H}$, d, $J=8,2 \mathrm{~Hz}$ ), 7,26 (1H, d, $J=8,2 \mathrm{~Hz}$ );. EM $70 \mathrm{eV}$ (ab. rel.) $\mathrm{m} / \mathrm{z}$ : Tabela 1 (letra $\mathbf{k})$.

\section{Cleistanta-1,8,11,13-tetraen-3,7-diona (6)}

IV $v_{\max }(\mathrm{KBr}) \mathrm{cm}^{-1} 2960,1700,1670,1465,1375,1110$, 1045,$815 ; \mathrm{RMN}^{1} \mathrm{H}\left(200 \mathrm{MHz}, \mathrm{CDCl}_{3}\right) \delta 1,08(3 \mathrm{H}, \mathrm{s}), 1,20$ $(3 \mathrm{H}, \mathrm{t}, J=7,4 \mathrm{~Hz}), 1,37(3 \mathrm{H}, \mathrm{s}), 1,52(3 \mathrm{H}, \mathrm{s}), 2,33(3 \mathrm{H}, \mathrm{s})$, $6,08(1 \mathrm{H}, \mathrm{d}, J=10,4 \mathrm{~Hz}), 7,04(1 \mathrm{H}, \mathrm{d}, J=8,1 \mathrm{~Hz}), 7,26(1 \mathrm{H}$, d, $J=8,1 \mathrm{~Hz}), 7,40(1 \mathrm{H}, \mathrm{d}, J=10,4 \mathrm{~Hz})$; EM $70 \mathrm{eV}$ (ab. rel.) $\mathrm{m} / \mathrm{z}$ : Tabela 1 (letra i).

\section{3- $\beta$-Acetóxi-oleano-12-eno (9a)}

$\mathrm{RMN}{ }^{1} \mathrm{H}\left(200 \mathrm{MHz}, \mathrm{CDCl}_{3}\right) \delta 0,84(6 \mathrm{H}, \mathrm{s}), 0,88(9 \mathrm{H}, \mathrm{s})$, $0,94(3 \mathrm{H}, \mathrm{s}), 0,97(3 \mathrm{H}, \mathrm{s}), 1,13(3 \mathrm{H}, \mathrm{s}), 2,04(3 \mathrm{H}, \mathrm{s}), 4,51(1 \mathrm{H}$, m), 5,18 (1H, t, $J=3,5 \mathrm{~Hz})$; EM $70 \mathrm{eV}$ (ab. rel.) m/z 468 (3), 218 (100), 203 (48), 189 (17), 175 (7), 164 (6), 147 (9), 135 (12), 119 (16), 109 (16), 95 (21), 81 (19), 69 (26), 55 (21).

\section{CONCLUSÃO}

Através da obtenção do perfil cromatográfico por CGAR e posterior uso de CGAR/EM para determinação do perfil químico, apoiado na análise de padrões de fragmentação, foram detectados, no extrato hexânico de Vellozia aff. carunculares, quatorze diterpenóides com esqueleto cleistantano e triterpenóides. $\mathrm{O}$ uso de métodos fitoquímicos clássicos levou ao isolamento de quatro dos cleistantanos detectados e um triterpenóide. 


\section{AGRADECIMENTOS}

Os autores agradecem ao Laboratório de Produtos NaturaisFIOCRUZ, pelos espectros de IV e RMN (200 MHz); ao Laboratório de Análise do DQI/IQ-UFRJ pelos espectros de IV; ao CESTEH/ENSP/FIOCRUZ e LADETEC IQ-UFRJ, pelos espectros de massas e ao CNPq/CEPG-UFRJ e CAPES, pelo apoio financeiro ao projeto.

\section{REFERÊNCIAS}

1. Menezes, N. L.; Linnean Society Symposium 1980, Series $\mathrm{n}^{\mathrm{o}} 8$, p. 117-138.

2. Desde 1978 até o momento, foram publicados cerca de 40 artigos envolvendo o isolamento de di e triterpenóides de Velloziaceae.

3. Branco, A.; Braz-Filho, R.; Kaiser, C. R.; Pinto, A. C.; Phytochemistry 1997, 47, 471.

4. Gunatilaka, A. A. L.; Kingston, D. G. J.; Johnson, R. R.; Pure Appl. Chem. 1994, 66, 2219.

5. Valente, L. M. M.; Gunatilaka, A. A. L.; Kingston, D. G. J.; Patitucci, M. L.; Pinto, A. C.; J. Nat. Prod. 1997, 60, 478.
6. Patitucci, M. L.; Veiga Júnior, V. F.; Pinto, A. C.; Quim. Nova 1995, 18, 262.

7. Budzikiewicz, H.; Wilson, J. M.; Djerassi, C.; J. Am. Chem. Soc. 1963, 85, 3688.

8. Enzell, C. R.; Tetrahedron Lett. 1966, 19, 2135.

9. Enzell, C. R.; Wahlberg, I.; Acta Chem. Scand. 1969, 23, 871.

10. Enzell, C. R.; Wahlberg, I.; Acta Chem. Scand. 1970, 24, 2498.

11. Enzell, C. R.; Ryhage, R.; Arkiv Kemi 1967, 27, 213.

12. Cleistantanos são diterpenos tricíclicos que podem ou não apresentar aromaticidade no anel $\mathrm{C}$. $\mathrm{Na}$ família Velloziaceae foram isolados vinte e um diterpenóides com esqueleto cleistantano, todos apresentando anel C aromá tico. Pinto, A. C.; Pereira, A. L.; Antunes, O. A. C.; Quim. Nova 1985, 8, 7.

13. Patitucci, M. L.; Tese MSc, Universidade Federal do Rio de Janeiro 1983.

14. Pinto, A. C.; Patitucci, M. L.; Silva, R. S.; Queiroz, P. P. S.; Kelecon, A.; Tetrahedron 1983, 39, 3351.

15. Pinto, A. C.; Epifanio, R. A.; Pizzolatti, M. G.; Phytochemistry 1992, 31, 1679.

16. Pinto, A. C.; Peixoto, E. M.; Fiorani, N. G. M.; Phytochemistry 1984, 23, 1293. 